\title{
Parity Theorems for Statistics on Domino Arrangements
}

\author{
Mark A. Shattuck \\ Mathematics Department \\ University of Tennessee \\ Knoxville, TN 37996-1300 \\ shattuck@math.utk.edu
}

\author{
Carl G. Wagner \\ Mathematics Department \\ University of Tennessee \\ Knoxville, TN 37996-1300 \\ wagner@math.utk.edu
}

Submitted: Oct 12, 2004; Accepted: Jan 21, 2005; Published: Jun 14, 2005

MR Subject Classifications: 11B39, 05A15

\begin{abstract}
We study special values of Carlitz's $q$-Fibonacci and $q$-Lucas polynomials $F_{n}(q, t)$ and $L_{n}(q, t)$. Brief algebraic and detailed combinatorial treatments are presented, the latter based on the fact that these polynomials are bivariate generating functions for a pair of statistics defined, respectively, on linear and circular domino arrangements.
\end{abstract}

\section{Introduction}

In what follows, $\mathbb{N}$ and $\mathbb{P}$ denote, respectively, the nonnegative and the positive integers. If $q$ is an indeterminate, then $n_{q}:=1+q+\cdots+q^{n-1}$ if $n \in \mathbb{P}, 0_{q}^{!}:=1, n_{q}^{!}:=1_{q} 2_{q} \cdots n_{q}$ if $n \in \mathbb{P}$, and

$$
\left(\begin{array}{l}
n \\
k
\end{array}\right)_{q}:= \begin{cases}\frac{n_{\dot{q}}^{!}}{k_{\dot{q}}^{!}(n-k) !}, & \text { if } 0 \leqslant k \leqslant n \\
0, & \text { if } k<0 \text { or } 0 \leqslant n<k .\end{cases}
$$

A useful variation of (1.1) is the well known formula [10, p.29]

$$
\left(\begin{array}{l}
n \\
k
\end{array}\right)_{q}=\sum_{\substack{d_{0}+d_{1}+\cdots+d_{k}=n-k \\
d_{i} \in \mathbb{N}}} q^{0 d_{0}+1 d_{1}+\cdots+k d_{k}}=\sum_{t \geqslant 0} p(k, n-k, t) q^{t}
$$

where $p(k, n-k, t)$ denotes the number of partitions of the integer $t$ with at most $n-k$ parts, each no larger than $k$.

This paper elucidates certain features of the $q$-Fibonacci polynomials

$$
F_{n}(q, t):=\sum_{0 \leqslant k \leqslant\lfloor n / 2\rfloor} q^{k^{2}}\left(\begin{array}{c}
n-k \\
k
\end{array}\right)_{q} t^{k}, \quad n \in \mathbb{N},
$$


and the $q$-Lucas polynomials

$$
L_{n}(q, t):=\sum_{0 \leqslant k \leqslant\lfloor n / 2\rfloor} q^{k^{2}} \frac{n_{q}}{(n-k)_{q}}\left(\begin{array}{c}
n-k \\
k
\end{array}\right)_{q} t^{k}, \quad n \in \mathbb{P} .
$$

Note that $F_{n}(1,1)=F_{n}$, where $F_{0}=F_{1}=1$ and $F_{n}=F_{n-1}+F_{n-2}, n \geqslant 2$ (this parameterization of the Fibonacci numbers, also employed by Wilf [12], results here in a notation with mnemonic features superior to that of the classical parameterization), and $L_{n}(1,1)=L_{n}$, where $L_{1}=1, L_{2}=3$, and $L_{n}=L_{n-1}+L_{n-2}, n \geqslant 3$. Our aim here is to present both algebraic and combinatorial treatments of $F_{n}(1,-1), F_{n}(-1, t), L_{n}(1,-1)$, and $L_{n}(-1, t)$.

Our algebraic proofs make frequent use of the identity [11, pp. 201-202]

$$
\sum_{n \geqslant 0}\left(\begin{array}{l}
n \\
k
\end{array}\right)_{q} x^{n}=\frac{x^{k}}{(1-x)(1-q x) \cdots\left(1-q^{k} x\right)}, \quad k \in \mathbb{N} .
$$

Our combinatorial proofs use the fact that $F_{n}(q, t)$ and $L_{n}(q, t)$ are generating functions for a pair of statistics defined, respectively, on linear and circular arrangements of nonoverlapping dominos, and embody the following general strategy:

Let $\left(\Gamma_{n}\right)$ be a sequence of finite discrete structures, with $\left|\Gamma_{n}\right|=G_{n}$. Each statistic $s: \bigcup \Gamma_{n} \rightarrow \mathbb{N}$ gives rise to a $q$-generalization of $G_{n}$, in the form of the generating function

$$
G_{n}(q):=\sum_{\gamma \in \Gamma_{n}} q^{s(\gamma)}=\sum_{k}\left|\left\{\gamma \in \Gamma_{n}: s(\gamma)=k\right\}\right| q^{k}
$$

Of course, $G_{n}(1)=G_{n}$. On the other hand,

$$
G_{n}(-1)=\left|\Gamma_{n}^{(0)}\right|-\left|\Gamma_{n}^{(1)}\right|
$$

where $\Gamma_{n}^{(i)}:=\left\{\gamma \in \Gamma_{n}: s(\gamma) \equiv i(\bmod 2)\right\}$. Thus a combinatorial proof that $G_{n}(-1)=g_{n}$ may be had by (1) identifying a distinguished subset $\Gamma_{n}^{*}$ of $\Gamma_{n}$ (with $\Gamma_{n}^{*}=\varnothing$ if $g_{n}=0$ and, more generally, $\left|\Gamma_{n}^{*}\right|=\left|g_{n}\right|$, with $\Gamma_{n}^{*}$ being a subset of $\Gamma_{n}^{(0)}$ or $\Gamma_{n}^{(1)}$, depending on whether $g_{n}$ is positive or negative), and (2) constructing an involution $\gamma \mapsto \gamma^{\prime}$ of $\Gamma_{n}-\Gamma_{n}^{*}$ for which $s(\gamma)$ and $s\left(\gamma^{\prime}\right)$ have opposite parity. (In what follows, we call the parity of $s(\gamma)$ the $s$-parity of $\gamma$, and the map $\gamma \mapsto \gamma^{\prime}$ an s-parity changing involution of $\left.\Gamma_{n}-\Gamma_{n}^{*}\right)$. In addition to conveying a visceral understanding of why $G_{n}(-1)$ takes its particular value, such an exercise furnishes a combinatorial proof of the congruence $G_{n} \equiv g_{n}(\bmod 2)$. Shattuck [9] has, for example, given such a combinatorial proof of the congruence

$$
S(n, k) \equiv\left(\begin{array}{c}
n-\lfloor k / 2\rfloor-1 \\
n-k
\end{array}\right) \quad(\bmod 2)
$$

for Stirling numbers of the second kind, answering a question posed by Stanley [10, p. 46, Exercise 17b]. 
The polynomials $F_{n}(q, t)$ and $L_{n}(q, t)$, or special cases thereof, have appeared previously in several guises. In a paper of Carlitz [1], $F_{n}(q, 1)$ arises as the generating function for the statistic $a_{1}+2 a_{2}+\cdots+(n-1) a_{n-1}$ on the set of binary words $a_{1} a_{2} \cdots a_{n-1}$ with no consecutive ones. In the same paper, $L_{n}(q, 1)$ occurs (though not explicitly in the simple form entailed by (1.4)) as the generating function for the statistic $a_{1}+2 a_{2}+\cdots+n a_{n}$ on the set of binary words $a_{1} a_{2} \cdots a_{n}$ with no consecutive ones, and with $a_{1}=a_{n}=1$ forbidden as well. Cigler [6] has shown that $F_{n}(q, t)$ arises as the bivariate generating function for a pair of statistics on the set of lattice paths from $(0,0)$ to $(n, 0)$ involving only horizontal moves, and northeast moves, followed immediately by southeast moves. Finally, Carlitz [2] has studied the $q$-Fibonacci polynomial $\Phi_{n}(a, q)=a^{n-1} F_{n-1}\left(q, a^{-2}\right)$ from a strictly algebraic point of view. See also the related paper of Cigler [3].

In $\S 2$ below, we treat the $q$-Fibonacci polynomials $F_{n}(q, t)$ and evaluate $F_{n}(1,-1)$ and $F_{n}(-1, t)$. In $\S 3$ we treat the $q$-Lucas polynomials $L_{n}(q, t)$ and evaluate $L_{n}(1,-1)$ and $L_{n}(-1, t)$. While the combinatorial proofs presented below could of course be reformulated in terms of the aforementioned statistics on binary words or lattice paths, our approach, based on statistics on domino arrangements, yields the most transparent constructions of the relevant parity changing involutions.

\section{Linear Domino Arrangements}

A well known problem of elementary combinatorics asks for the number of ways to place $k$ indistinguishable non-overlapping dominos on the numbers $1,2, \ldots, n$, arranged in a row, where a domino is a rectangular piece capable of covering two numbers. It is useful to place squares (pieces covering a single number) on each number not covered by a domino. The original problem then becomes one of determining the cardinality of $\mathcal{R}_{n, k}$, the set of coverings of the row of numbers $1,2, \ldots, n$ by $k$ dominos and $n-2 k$ squares. Since each such covering corresponds uniquely to a word in the alphabet $\{d, s\}$ comprising $k d$ 's and $n-2 k s$ 's, it follows that

$$
\left|\mathcal{R}_{n, k}\right|=\left(\begin{array}{c}
n-k \\
k
\end{array}\right), \quad 0 \leqslant k \leqslant n / 2,
$$

for all $n \in \mathbb{P}$. (In what follows we will simply identify coverings with such words.) If we set $\mathcal{R}_{0,0}=\{\varnothing\}$, the "empty covering," then (2.1) holds for $n=0$ as well. With

$$
\mathcal{R}_{n}:=\bigcup_{0 \leqslant k \leqslant\lfloor n / 2\rfloor} \mathcal{R}_{n, k}, \quad n \in \mathbb{N},
$$

it follows that

$$
\left|\mathcal{R}_{n}\right|=\sum_{0 \leqslant k \leqslant\lfloor n / 2\rfloor}\left(\begin{array}{c}
n-k \\
k
\end{array}\right)=F_{n},
$$

where $F_{0}=F_{1}=1$ and $F_{n}=F_{n-1}+F_{n-2}$ for $n \geqslant 2$. 
Given $c \in \mathcal{R}_{n}$, let $\nu(c):=$ the number of dominos in the covering $c$, let $\sigma(c):=$ the sum of the numbers covered by the left halves of each of those dominos, and let

$$
F_{n}(q, t):=\sum_{c \in \mathcal{R}_{n}} q^{\sigma(c)} t^{\nu(c)}
$$

Categorizing covers of $1,2, \ldots, n$ according as $n$ is covered by a square or a domino yields the recurrence relation

$$
F_{n}(q, t)=F_{n-1}(q, t)+q^{n-1} t F_{n-2}(q, t), \quad n \geqslant 2,
$$

with $F_{0}(q, t)=F_{1}(q, t)=1$. The following theorem gives an explicit formula for $F_{n}(q, t)$.

Theorem 2.1. For all $n \in \mathbb{N}$,

$$
F_{n}(q, t)=\sum_{0 \leqslant k \leqslant\lfloor n / 2\rfloor} q^{k^{2}}\left(\begin{array}{c}
n-k \\
k
\end{array}\right)_{q} t^{k} .
$$

Proof. It clearly suffices to show that

$$
\sum_{c \in \mathcal{R}_{n, k}} q^{\sigma(c)}=q^{k^{2}}\left(\begin{array}{c}
n-k \\
k
\end{array}\right)_{q}
$$

Each $c \in \mathcal{R}_{n, k}$ corresponds uniquely to a sequence $\left(d_{0}, d_{1}, \ldots, d_{k}\right)$, where $d_{0}$ is the number of squares following the $k^{\text {th }}$ domino (counting from left to right) in the covering $c, d_{k}$ is the number of squares preceding the first domino, and, for $0<i<k, d_{k-i}$ is the number of squares between dominos $i$ and $i+1$. Here, $\sigma(c)=\left(d_{k}+1\right)+\left(d_{k}+d_{k-1}+3\right)+\cdots+$ $\left(d_{k}+d_{k-1}+\cdots+d_{1}+(2 k-1)\right)=k^{2}+0 d_{0}+1 d_{1}+2 d_{2}+\cdots+k d_{k}$. Hence,

$$
\sum_{c \in \mathcal{R}_{n, k}} q^{\sigma(c)}=q^{k^{2}} \sum_{\substack{d_{0}+d_{1}+\cdots+d_{k}=n-2 k \\
d_{i} \in \mathbb{N}}} q^{0 d_{0}+1 d_{1}+\cdots+k d_{k}}=q^{k^{2}}\left(\begin{array}{c}
n-k \\
k
\end{array}\right)_{q}
$$

by (1.2).

Corollary 2.1.1. The ordinary generating function of the sequence $\left(F_{n}(q, t)\right)_{n \geqslant 0}$ is given by

$$
\sum_{n \geqslant 0} F_{n}(q, t) x^{n}=\sum_{k \geqslant 0} \frac{q^{k^{2}} t^{k} x^{2 k}}{(1-x)(1-q x) \cdots\left(1-q^{k} x\right)} .
$$

Proof. The result follows from (2.6), summation interchange, and (1.5).

As noted earlier, $F_{n}(1,1)=F_{n}$. Hence $(2.7)$ generalizes the well known result,

$$
\sum_{n \geqslant 0} F_{n} x^{n}=\frac{1}{1-x-x^{2}}
$$

We now evaluate $F_{n}(1,-1)$ and $F_{n}(-1, t)$.

Substituting $(q, t)=(1,-1)$ into $(2.5)$ and solving the resulting recurrence yields 
Theorem 2.2. For all $n \in \mathbb{N}$,

$$
F_{n}(1,-1)=\left\{\begin{array}{lll}
0, & \text { if } n \equiv 2 \text { or } 5 & (\bmod 6) \\
1, & \text { if } n \equiv 0 \text { or } 1 & (\bmod 6) \\
-1, & \text { if } n \equiv 3 \text { or } 4 & (\bmod 6)
\end{array}\right.
$$

A slight variation on the strategy outlined in $\S 1$ above yields a combinatorial proof of (2.9). Let $\mathcal{R}_{n}^{*}$ consist of those $c=x_{1} x_{2} \cdots$ in $\mathcal{R}_{n}$ satisfying the conditions $x_{2 i-1} x_{2 i}=d s$, $1 \leqslant i \leqslant\lfloor n / 3\rfloor$. If $n \equiv 0$ or $1(\bmod 6)$, then $\mathcal{R}_{n}^{*}$ is a singleton whose sole element has even $\nu$-parity. If $n \equiv 3$ or $4(\bmod 6)$, then $\mathcal{R}_{n}^{*}$ is a singleton whose sole element has odd $\nu$-parity. If $n \equiv 2(\bmod 3)$, then $\mathcal{R}_{n}^{*}$ is a doubleton containing two members of opposite $\nu$-parity, which we pair. The foregoing observations establish (2.9) for $0 \leqslant n \leqslant 2$ since $\mathcal{R}_{n}^{*}=\mathcal{R}_{n}$ for such $n$. Thus, it remains only to construct a $\nu$-parity changing involution of $\mathcal{R}_{n}-\mathcal{R}_{n}^{*}$ for $n \geqslant 3$. Such an involution is furnished by the pairings

$$
(d s)^{k} s d v \leftrightarrow(d s)^{k} s s s v
$$

and

$$
(d s)^{k} d d u \leftrightarrow(d s)^{k} s s d u
$$

where $0 \leqslant k<\lfloor n / 3\rfloor,(d s)^{0}$ denotes the empty word, and $u$ and $v$ are (possibly empty) words in the alphabet $\{d, s\}$. Note that the above argument also furnishes a combinatorial proof of the well known fact that $F_{n}$ is even if and only if $n \equiv 2(\bmod 3)$.

Remark. Neither (2.9), nor its corollary (3.12) below, is new. Indeed, (2.9) is a special case of the well known formula

$$
\sum_{0 \leqslant k \leqslant\lfloor n / 2\rfloor}(-1)^{k} q^{\left(\begin{array}{c}
k \\
2
\end{array}\right)}\left(\begin{array}{c}
n-k \\
k
\end{array}\right)_{q}= \begin{cases}(-1)^{\lfloor n / 3\rfloor} q^{n(n-1) / 6}, & \text { if } n \equiv 0,1 \quad(\bmod 3) ; \\
0, & \text { if } n \equiv 2 \quad(\bmod 3) .\end{cases}
$$

See, e.g., Ekhad and Zeilberger [7], Kupershmidt [8], and Cigler [4]. Our interest here, and in Theorem 3.2 below, has been to furnish new proofs of (2.9) and (3.12) based on parity changing involutions.

Theorem 2.3. For all $m \in \mathbb{N}$,

$$
F_{2 m}(-1, t)=F_{m}\left(1, t^{2}\right)-t F_{m-1}\left(1, t^{2}\right)
$$

and

$$
F_{2 m+1}(-1, t)=F_{m}\left(1, t^{2}\right)
$$

where $F_{-1}(q, t):=0$. 
Proof. Taking the even and odd parts of both sides of (2.7) and replacing $x$ with $x^{1 / 2}$ yields

$$
\sum_{m \geqslant 0} F_{2 m}(-1, t) x^{m}=(1-t x) \sum_{k \geqslant 0} \frac{t^{2 k} x^{2 k}}{(1-x)^{k+1}}
$$

and

$$
\sum_{m \geqslant 0} F_{2 m+1}(-1, t) x^{m}=\sum_{k \geqslant 0} \frac{t^{2 k} x^{2 k}}{(1-x)^{k+1}},
$$

from which (2.10) and (2.11) follow from (2.7).

For a combinatorial proof of (2.10) and (2.11), we first assign to each domino arrangement $c \in \mathcal{R}_{n}$ the weight $w_{c}:=(-1)^{\sigma(c)} t^{v(c)}$, where $t$ is an indeterminate. Let $\mathcal{R}_{n}^{\prime}$ consist of those $c=x_{1} x_{2} \cdots x_{r}$ in $\mathcal{R}_{n}$ satisfying the conditions $x_{2 i-1}=x_{2 i}, 1 \leqslant i \leqslant\lfloor r / 2\rfloor$. Suppose $c=x_{1} x_{2} \cdots x_{r} \in \mathcal{R}_{n}-\mathcal{R}_{n}^{\prime}$, with $i_{0}$ being the smallest value of $i$ for which $x_{2 i-1} \neq x_{2 i}$. Exchanging the positions of $x_{2 i_{0}-1}$ and $x_{2 i_{0}}$ within $c$ produces a $\sigma$-parity changing involution of $\mathcal{R}_{n}-\mathcal{R}_{n}^{\prime}$ which preserves $v(c)$. Then

$$
F_{2 m+1}(-1, t)=\sum_{c \in \mathcal{R}_{2 m+1}} w_{c}=\sum_{c \in \mathcal{R}_{2 m+1}^{\prime}} w_{c}=\sum_{s \in \mathcal{R}_{m}} w_{s}^{2}=F_{m}\left(1, t^{2}\right)
$$

and

$$
\begin{aligned}
F_{2 m}(-1, t) & =\sum_{c \in \mathcal{R}_{2 m}} w_{c}=\sum_{c \in \mathcal{R}_{2 m}^{\prime}} w_{c}=\sum_{\substack{c \in \mathcal{R}_{2 m}^{\prime} \\
\sigma(c) \text { even }}} w_{c}+\sum_{\substack{c \in \mathcal{R}_{2 m}^{\prime} \\
\sigma(c) \text { odd }}} w_{c} \\
& =\sum_{s \in \mathcal{R}_{m}} w_{s}^{2}-t \sum_{s \in \mathcal{R}_{m-1}} w_{s}^{2}=F_{m}\left(1, t^{2}\right)-t F_{m-1}\left(1, t^{2}\right),
\end{aligned}
$$

since members of $\mathcal{R}_{2 m+1}^{\prime}$ end in a single $s$, while members of $\mathcal{R}_{2 m}^{\prime}$ end in a double letter or in a single $d$, depending on whether $\sigma(c)$ is even or odd.

When $t=1$ in Theorem 2.3, we get for $m \in \mathbb{N}$,

$$
F_{2 m}(-1,1)=F_{m-2} \quad \text { and } \quad F_{2 m+1}(-1,1)=F_{m}
$$

The arguments given above then specialize when $t=1$ to furnish combinatorial proofs of the congruences $F_{2 m} \equiv F_{m-2}(\bmod 2)$ and $F_{2 m+1} \equiv F_{m}(\bmod 2)$.

\section{Circular Domino Arrangements}

If $n \in \mathbb{P}$ and $0 \leqslant k \leqslant\lfloor n / 2\rfloor$, let $\mathcal{C}_{n, k}$ be the set of coverings by $k$ dominos and $n-2 k$ squares of the numbers $1,2, \ldots, n$ arranged clockwise around a circle: 


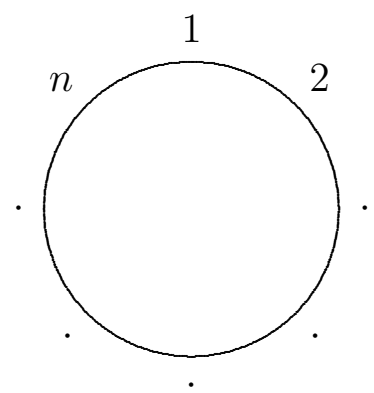

By the initial half of a domino occurring in such a cover, we mean the half first encountered as the circle is traversed clockwise. Classifying members of $\mathcal{C}_{n, k}$ according as (i) $n$ is covered by the initial half of some domino, (ii) 1 is covered by the initial half of some domino, or (iii) 1 is covered by a square, and applying (2.1) to count these three classes yields the well known result

$$
\left|\mathcal{C}_{n, k}\right|=2\left(\begin{array}{c}
n-k-1 \\
k-1
\end{array}\right)+\left(\begin{array}{c}
n-k-1 \\
k
\end{array}\right)=\frac{n}{n-k}\left(\begin{array}{c}
n-k \\
k
\end{array}\right), \quad 0 \leqslant k \leqslant\lfloor n / 2\rfloor .
$$

Note that $\left|\mathcal{C}_{2,1}\right|=2$, the relevant coverings being

(1)

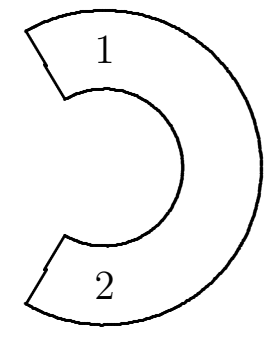

and

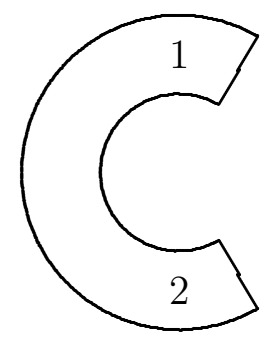

In covering (1), the initial half of the domino covers 1 , and in covering (2), the initial half covers 2 .

With

$$
\mathcal{C}_{n}:=\bigcup_{0 \leqslant k \leqslant\lfloor n / 2\rfloor} \mathcal{C}_{n, k}, \quad n \in \mathbb{P}
$$

it follows that

$$
\left|\mathcal{C}_{n}\right|=\sum_{0 \leqslant k \leqslant\lfloor n / 2\rfloor} \frac{n}{n-k}\left(\begin{array}{c}
n-k \\
k
\end{array}\right)=L_{n},
$$

where $L_{1}=1, L_{2}=3$, and $L_{n}=L_{n-1}+L_{n-2}$ for $n \geqslant 2$, also a well known result.

Given $c \in \mathcal{C}_{n}$, let $\nu(c):=$ the number of dominos in the covering $c$, let $\sigma(c):=$ the sum of the numbers covered by the initial halves of each of those dominos, and let

$$
L_{n}(q, t):=\sum_{c \in \mathcal{C}_{n}} q^{\sigma(c)} t^{\nu(c)}
$$


Theorem 3.1. For all $n \in \mathbb{P}$,

$$
L_{n}(q, t)=\sum_{0 \leqslant k \leqslant\lfloor n / 2\rfloor} q^{k^{2}} \frac{n_{q}}{(n-k)_{q}}\left(\begin{array}{c}
n-k \\
k
\end{array}\right)_{q} t^{k} .
$$

Proof. It suffices to show that

$$
\sum_{c \in \mathcal{C}_{n, k}} q^{\sigma(c)}=q^{k^{2}} \frac{n_{q}}{(n-k)_{q}}\left(\begin{array}{c}
n-k \\
k
\end{array}\right)_{q}
$$

for $0 \leqslant k \leqslant\lfloor n / 2\rfloor$. Partitioning $\mathcal{C}_{n, k}$ into the categories (i), (ii), and (iii) employed above in deriving (3.1), and applying (2.6) yields

$$
\begin{aligned}
\sum_{c \in \mathcal{C}_{n, k}} q^{\sigma(c)} & =q^{k^{2}-k+n}\left(\begin{array}{c}
n-k-1 \\
k-1
\end{array}\right)_{q}+q^{k^{2}}\left(\begin{array}{c}
n-k-1 \\
k-1
\end{array}\right)_{q}+q^{k^{2}+k}\left(\begin{array}{c}
n-k-1 \\
k
\end{array}\right)_{q} \\
& =q^{k^{2}} \frac{n_{q}}{(n-k)_{q}}\left(\begin{array}{c}
n-k \\
k
\end{array}\right)_{q} .
\end{aligned}
$$

Corollary 3.1.1. The ordinary generating function of the sequence $\left(L_{n}(q, t)\right)_{n \geqslant 1}$ is given by

$$
\sum_{n \geqslant 1} L_{n}(q, t) x^{n}=\frac{x}{1-x}+\sum_{k \geqslant 1} \frac{q^{k^{2}} t^{k} x^{2 k}\left(1+q^{k}(1-x)\right)}{(1-x)(1-q x) \cdots\left(1-q^{k} x\right)} .
$$

Proof. This result follows from (3.5), using the identity

$$
\frac{n_{q}}{(n-k)_{q}}\left(\begin{array}{c}
n-k \\
k
\end{array}\right)_{q}=\left(\begin{array}{c}
n-k \\
k
\end{array}\right)_{q}+q^{n-k}\left(\begin{array}{c}
n-k-1 \\
k-1
\end{array}\right)_{q},
$$

summation interchange, and (1.5).

As noted earlier, $L_{n}(1,1)=L_{n}$. Hence (3.7) generalizes the well known result

$$
\sum_{n \geqslant 1} L_{n} x^{n}=\frac{x+2 x^{2}}{1-x-x^{2}} .
$$

The $L_{n}(q, t)$ are related to the $F_{n}(q, t)$ by the formula

$$
L_{n}(1, t)=F_{n}(1, t)+t F_{n-2}(1, t), \quad n \geqslant 1,
$$

which reduces to the familiar

$$
L_{n}=F_{n-1}+2 F_{n-2}, \quad n \geqslant 1,
$$

when $t=1$. We now evaluate $L_{n}(1,-1)$ and $L_{n}(-1, t)$.

Substituting $t=-1$ into (3.10) and applying (2.9) yields 
Theorem 3.2. For all $n \in \mathbb{P}$,

$$
L_{n}(1,-1)= \begin{cases}1, & \text { if } n \equiv 1 \text { or } 5 \quad(\bmod 6) \\ -1, & \text { if } n \equiv 2 \text { or } 4 \quad(\bmod 6) \\ 2, & \text { if } n \equiv 0 \quad(\bmod 6) \\ -2, & \text { if } n \equiv 3 \quad(\bmod 6)\end{cases}
$$

For a combinatorial proof of $(3.12)$, write $\mathcal{C}_{n}=\overrightarrow{\mathcal{C}}_{n} \cup \overleftarrow{\mathcal{C}}_{n}$, where $c \in \overrightarrow{\mathcal{C}}_{n}$ iff 1 is covered by a square of $c$, or 1 and 2 by a single domino of $c$, and $c \in \overleftarrow{\mathcal{C}}_{n}$ iff $n$ and 1 are covered by a single domino of $c$. Associate to each $c \in \mathcal{C}_{n}$ a word $u_{c}=v_{1} v_{2} \cdots$ in the alphabet $\{d, s\}$, where

$$
v_{i}:= \begin{cases}s, & \text { if the " } i \text {-th piece" of } c \text { is a square; } \\ d, & \text { if the " } i \text {-th piece" of } c \text { is a domino, }\end{cases}
$$

and one determines the " $i$-th piece" of $c$ by starting at 1 and proceeding clockwise if $c \in \overrightarrow{\mathcal{C}}_{n}$, and counterclockwise if $c \in \overleftarrow{\mathcal{C}}_{n}$. Note that although distinct elements of $\mathcal{C}_{n}$ may be associated with the same word, each $c \in \overrightarrow{\mathcal{C}}_{n}$ is associated with a unique word, and each $c \in \overleftarrow{\mathcal{C}}_{n}$ is associated with a unique word.

Let

$$
\begin{aligned}
& \mathcal{C}_{n}^{*}:=\left\{c \in \overrightarrow{\mathcal{C}}_{n}: u_{c}=(s d)^{\lfloor n / 3\rfloor} \text { or }(s d)^{\lfloor n / 3\rfloor} s\right\} \\
& \cup\left\{c \in \overleftarrow{\mathcal{C}}_{n}: u_{c}=(d s)^{\lfloor n / 3\rfloor} \text { or }(d s)^{\lfloor n / 3\rfloor} d\right\}
\end{aligned}
$$

It is straightforward to check that $\left|\mathcal{C}_{n}^{*}\right|=1$ if $n \equiv 1$ or $2(\bmod 3)$ and $\left|\mathcal{C}_{n}^{*}\right|=2$ if $n \equiv 0$ $(\bmod 3) . \quad$ In the former case, the sole element of $\mathcal{C}_{n}^{*}$ has even $\nu$-parity if $n \equiv 1$ or 5 $(\bmod 6)$, and odd $\nu$-parity if $n \equiv 2$ or $4(\bmod 6)$. In the latter case, both elements of $\mathcal{C}_{n}^{*}$ have even $\nu$-parity if $n \equiv 0(\bmod 6)$, and odd $\nu$-parity if $n \equiv 3(\bmod 6)$.

To complete the proof it suffices to identify a $\nu$-parity changing involution of $\overrightarrow{\mathcal{C}}_{n}-\mathcal{C}_{n}^{*}$, and of $\overleftarrow{\mathcal{C}}_{n}-\mathcal{C}_{n}^{*}$, whenever these sets are nonempty. In the former case, this occurs when $n \geqslant 2$, and such an involution is furnished by the pairing

$$
(s d)^{k} d u \leftrightarrow(s d)^{k} s s u
$$

where $0 \leqslant k \leqslant\lfloor(n-2) / 3\rfloor$, and $u$ is a (possibly empty) word in $\{d, s\}$. In the latter case, this occurs when $n \geqslant 4$, and such an involution is furnished by the pairing

$$
d(s d)^{j} d v \leftrightarrow d(s d)^{j} s s v
$$

where $0 \leqslant j \leqslant\lfloor(n-4) / 3\rfloor$, and $v$ is a (possibly empty) word in $\{d, s\}$.

Theorem 3.3. For all $m \in \mathbb{P}$,

$$
L_{2 m}(-1, t)=L_{m}\left(1, t^{2}\right)
$$


and

$$
L_{2 m-1}(-1, t)=F_{m-1}\left(1, t^{2}\right)-t F_{m-2}\left(1, t^{2}\right),
$$

where $F_{-1}(q, t):=0$.

Proof. Taking the even and odd parts of both sides of (3.7) and replacing $x$ with $x^{1 / 2}$ yields

$$
\sum_{m \geqslant 1} L_{2 m}(-1, t) x^{m}=\frac{x}{1-x}+(2-x) \sum_{k \geqslant 1} \frac{t^{2 k} x^{2 k}}{(1-x)^{k+1}}
$$

and

$$
\sum_{m \geqslant 1} L_{2 m-1}(-1, t) x^{m}=(1-t x) \sum_{k \geqslant 0} \frac{t^{2 k} x^{2 k+1}}{(1-x)^{k+1}},
$$

from which (3.13) and (3.14) follow from (3.7) and (2.7).

The following observation leads to a combinatorial proof of (3.13) and (3.14) for $n \geqslant 2$ : If $c \in \mathcal{C}_{n}$, let $c^{\prime}$ be the result of reflecting the arrangement of dominos and squares constituting $c$ in the diameter through the point 2 on the relevant circle. We illustrate pairs $c$ and $c^{\prime}$ for $n=8$ and $n=9$ below.
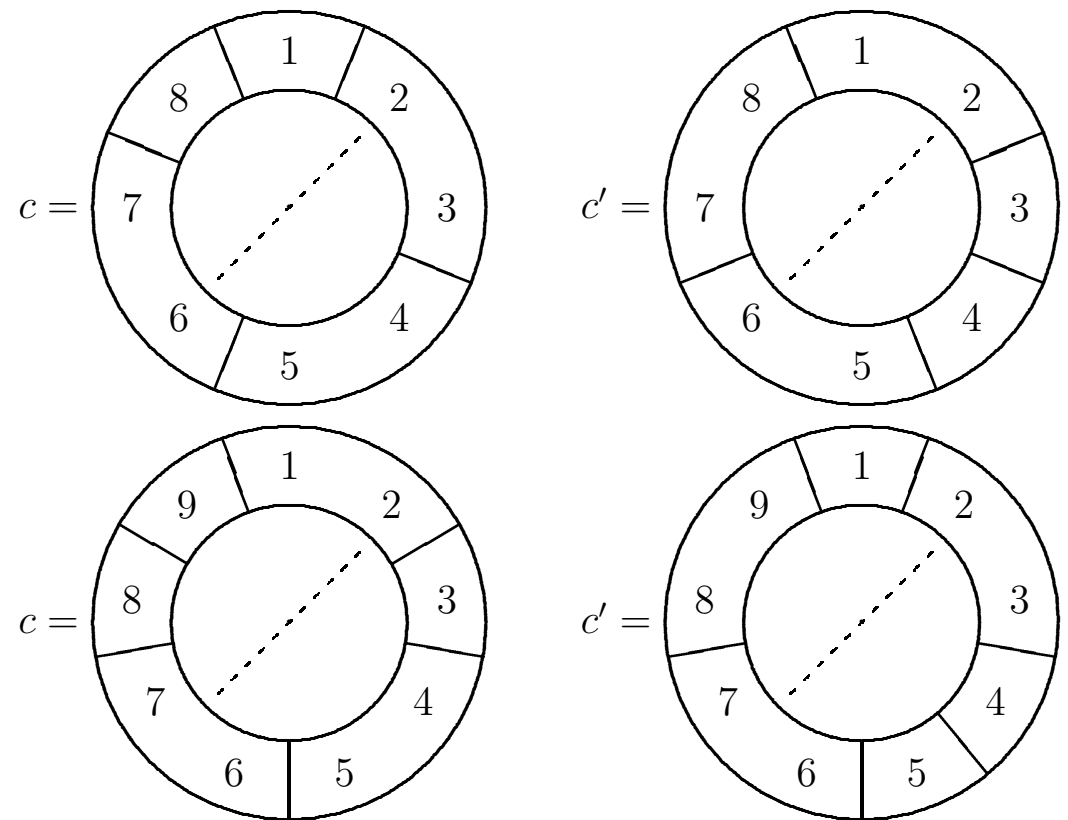

Note that $c$ and $c^{\prime}$ have opposite $\sigma$-parity in both cases. More generally, it may be verified that if $n$ is even, then $c$ and $c^{\prime}$ have opposite $\sigma$-parity iff $\nu(c)$ is odd, and if $n$ is odd, then $c$ and $c^{\prime}$ have opposite $\sigma$-parity iff 2 is covered by a domino in $c$.

In what follows we use the same encoding of covers as words in $\{d, s\}$ that we employed in the combinatorial proof of Theorem 3.2. We also assign to each $c \in \mathcal{C}_{n}$ the weight $w_{c}=(-1)^{\sigma(c)} t^{v(c)}$. 
Suppose that $n=2 m$. Let $\mathcal{C}_{2 m}^{\prime}:=\bigcup_{0 \leqslant k \leqslant m} \mathcal{C}_{2 m, 2 k}$ and let $\mathcal{C}_{2 m}^{*}$ consist of those $c \in \mathcal{C}_{2 m}^{\prime}$ for which $u_{c}=v_{1} v_{2} \cdots$ satisfies $v_{2 i-1}=v_{2 i}$ for all $i$. We extend the $\sigma$-parity changing, $v$-preserving involution of $\mathcal{C}_{2 m}-\mathcal{C}_{2 m}^{\prime}$ defined in our initial observation to $\mathcal{C}_{2 m}-\mathcal{C}_{2 m}^{*}$. Since $\mathcal{C}_{2 m}^{*}=\mathcal{C}_{2 m}^{\prime}$ for $m \leqslant 2$, we may restrict attention to the case $m \geqslant 3$. Let $c \in \mathcal{C}_{2 m}^{\prime}-\mathcal{C}_{2 m}^{*}$, with $u_{c}=v_{1} v_{2} \cdots$, and let $i_{0}$ be the largest $i$ for which $v_{2 i-1} \neq v_{2 i}$, whence $i_{0} \geqslant 2$. Interchanging the $\left(2 i_{0}-1\right)^{\text {th }}$ and $\left(2 i_{0}\right)^{\text {th }}$ pieces of $c$ furnishes such an involution. Then

$$
L_{2 m}(-1, t)=\sum_{c \in \mathcal{C}_{2 m}} w_{c}=\sum_{c \in \mathcal{C}_{2 m}^{*}} w_{c}=L_{m}\left(1, t^{2}\right) .
$$

Suppose now that $n=2 m-1$, where $m \geqslant 2$. Let $\mathcal{C}_{2 m-1}^{\prime}$ consist of those $c \in \mathcal{C}_{2 m-1}$ in which 2 is covered by a square. There is an obvious $v$-preserving bijection $b: \mathcal{C}_{2 m-1}^{\prime} \rightarrow$ $\mathcal{R}_{2 m-2}$ for which $\sigma(c) \equiv \sigma(b(c))(\bmod 2)$ for all $c \in \mathcal{C}_{2 m-1}^{\prime}$. In view of the involution of $\mathcal{C}_{2 m-1}-\mathcal{C}_{2 m-1}^{\prime}$ defined in our initial observation, we have

$$
\begin{aligned}
L_{2 m-1}(-1, t) & =\sum_{c \in \mathcal{C}_{2 m-1}} w_{c}=\sum_{c \in \mathcal{C}_{2 m-1}^{\prime}} w_{c}=\sum_{c \in \mathcal{R}_{2 m-2}} w_{c} \\
& =F_{m-1}\left(1, t^{2}\right)-t F_{m-2}\left(1, t^{2}\right),
\end{aligned}
$$

by $(2.10)$.

When $t=1$ in Theorem 3.3, we get for $m \in \mathbb{P}$,

$$
L_{2 m}(-1,1)=L_{m} \quad \text { and } \quad L_{2 m-1}(-1,1)=F_{m-3} .
$$

The arguments given above then specialize when $t=1$ to furnish combinatorial proofs of the congruences $L_{2 m} \equiv L_{m}(\bmod 2)$ and $L_{2 m-1} \equiv F_{m-3}(\bmod 2)$.

\section{Some Concluding Remarks}

Formulas such as (2.10), (2.11), (3.13), and (3.14) show that the $q=-1$ case differs in many respects from the general $q$-case. The referee points out to us that the reason for this behavior may lie in the fact that the Fibonacci and Lucas polynomials can be written as a sum of $q$-binomial coefficients, which are known to reduce to the ordinary binomial coefficients when $q=-1$. Similar reductions also occur in some cases when $q$ is a root of unity, and analogues to formulas such as (2.10) and (3.13) might be expected in these cases. For example, when $q=\rho=\frac{-1+i \sqrt{3}}{2}$, a third root of unity, we have the following formulas:

$$
\begin{aligned}
F_{3 m}(\rho, t) & =F_{m}\left(1, t^{3}\right)-t F_{m-1}\left(1, t^{3}\right), \\
F_{3 m+1}(\rho, t) & =F_{m}\left(1, t^{3}\right)+\rho t^{2} F_{m-1}\left(1, t^{3}\right), \\
F_{3 m+2}(\rho, t) & =(1+\rho t) F_{m}\left(1, t^{3}\right), \\
L_{3 m}(\rho, t) & =L_{m}\left(1, t^{3}\right), \\
L_{3 m+1}(\rho, t) & =F_{m}\left(1, t^{3}\right)+\rho t(1-\rho t) F_{m-1}\left(1, t^{3}\right),
\end{aligned}
$$


and

$$
L_{3 m+2}(\rho, t)=(1-t) F_{m}\left(1, t^{3}\right)-t^{2} F_{m-1}\left(1, t^{3}\right) .
$$

The combinatorial arguments given when $q=-1$ can be extended to identities such as these. For example, to prove (4.1)-(4.3), instead of pairing members of $\mathcal{R}_{n}$ with the same number of dominos and opposite $\sigma$-parity, we partition $\mathcal{R}_{n}$ into tripletons whose members each contain the same number of dominos but have different $\sigma$-values mod 3 . Assign to each member $c$ of $\mathcal{R}_{n}$ the weight $w_{c}:=\rho^{\sigma(c)} t^{v(c)}$, where $\rho=\frac{-1+i \sqrt{3}}{2}$ and $t$ is an indeterminate. Then for a tripleton $\left\{c_{1}, c_{2}, c_{3}\right\}$ as described, we have $w_{c_{1}}+w_{c_{2}}+w_{c_{3}}=0$ since $1+\rho+\rho^{2}=0$.

Let $\mathcal{R}_{n}^{\prime}$ consist of those $c=x_{1} x_{2} \cdots x_{r}$ in $\mathcal{R}_{n}$ satisfying

$$
x_{3 i-2}=x_{3 i-1}=x_{3 i}, \quad 1 \leqslant i \leqslant\lfloor r / 3\rfloor .
$$

Suppose that $c=x_{1} x_{2} \cdots x_{r} \in \mathcal{R}_{n}-\mathcal{R}_{n}^{\prime}$, with $i_{0}$ being the smallest value of $i$ for which (4.7) fails to hold. Group the three members of $\mathcal{R}_{n}-\mathcal{R}_{n}^{\prime}$ gotten by circularly permuting $x_{3 i_{0}-2}, x_{3 i_{0}-1}$, and $x_{3 i_{0}}$ within $c=x_{1} x_{2} \cdots x_{r}$, leaving the rest of $c$ undisturbed. Note that these three members of $\mathcal{R}_{n}$ contain the same number of dominos but have different $\sigma$-values mod 3 .

If $n=3 m$, then

$$
\begin{array}{r}
F_{3 m}(\rho, t)=\sum_{c \in \mathcal{R}_{3 m}} w_{c}=\sum_{c \in \mathcal{R}_{3 m}^{\prime}} w_{c}=\sum_{s \in \mathcal{R}_{m}} w_{s}^{3}+\left(\rho+\rho^{2}\right) t \sum_{s \in \mathcal{R}_{m-1}} w_{s}^{3}=F_{m}\left(1, t^{3}\right) \\
+\left(\rho+\rho^{2}\right) t F_{m-1}\left(1, t^{3}\right)=F_{m}\left(1, t^{3}\right)-t F_{m-1}\left(1, t^{3}\right),
\end{array}
$$

which proves (4.1), since members of $\mathcal{R}_{3 m}^{\prime}$ may end in a triple letter, in $-d s$, or in $-s d$. Formulas (4.2) and (4.3) follow similarly, since members of $\mathcal{R}_{3 m+1}^{\prime}$ end in $-s$ or $-d d$, while members of $\mathcal{R}_{3 m+2}^{\prime}$ end in $-s s$ or $-d$.

We conclude by remarking that Cigler has studied the generalized $q$-Fibonacci polynomials [5]

$$
F_{n}(j, s, t, q):=\sum_{0 \leqslant k j \leqslant n-j+1} q^{j\left(\begin{array}{c}
k \\
2
\end{array}\right)}\left(\begin{array}{c}
n-(j-1)(k+1) \\
k
\end{array}\right) t_{q}^{k} s^{n-(k+1) j+1} .
$$

Note that a close variant of (4.8),

$$
F_{n}^{(j)}(q, t, s):=F_{n+j-1}(j, s, q t, q), \quad n \in \mathbb{N},
$$

furnishes a natural generalization of $F_{n}(q, t)$, reducing to the latter when $j=2$ and $s=1$. Analogues of $(2.10)$ and $(2.11)$ can be obtained for $F_{n}^{(j)}(q, t, 1)$ when $j \geqslant 3$; for example, we have for $m \in \mathbb{N}$,

$$
\begin{aligned}
F_{2 m}^{(3)}(-1, t, 1) & =F_{m}^{(3)}\left(1,-t^{2}, 1\right), \\
F_{2 m+1}^{(3)}(-1, t, 1) & =F_{m}^{(3)}\left(1,-t^{2}, 1\right)-t F_{m-1}^{(3)}\left(1,-t^{2}, 1\right), \\
F_{2 m}^{(4)}(-1, t, 1) & =F_{m}^{(4)}\left(1, t^{2}, 1\right)-t F_{m-2}^{(4)}\left(1, t^{2}, 1\right),
\end{aligned}
$$


and

$$
F_{2 m+1}^{(4)}(-1, t, 1)=F_{m}^{(4)}\left(1, t^{2}, 1\right) .
$$

It would be interesting to have combinatorial proofs of (4.10)-(4.13).

\section{Acknowledgment}

The authors would like to thank the anonymous referee for a prompt, thorough reading of this paper and for many insightful suggestions which improved it. We are indebted to the referee for formulas (4.1)-(4.3), formulas (4.10)-(4.13), and for Theorems 2.3 and 3.3 in their given form. We also would like to thank the referee for calling to our attention the papers of Cigler [4, 5], Ekhad and Zeilberger [7], and Kupershmidt [8].

\section{References}

[1] L. Carlitz, Fibonacci notes 3: q-Fibonacci numbers, Fib. Quart. 12 (1974), 317-322.

[2] L. Carlitz, Fibonacci notes 4: q-Fibonacci polynomials, Fib. Quart. 13 (1975), 97102.

[3] J. Cigler, q-Fibonacci polynomials, Fib. Quart. 41 (2003), 31-40.

[4] J. Cigler, A new class of q-Fibonacci polynomials, Elect. J. Combin. 10 (2003), \#R19.

[5] J. Cigler, Some algebraic aspects of Morse code sequences, Discrete Math. and Theoretical Computer Science 6 (2003), 55-68.

[6] J. Cigler, $q$-Fibonacci polynomials and the Rogers-Ramanujan identities, Ann. Combin. 8 (2004), 269-285.

[7] S. Ekhad and D. Zeilberger, The number of solutions of $X^{2}=0$ in triangular matrices over $G F(q)$, Elect. J. Combin. 3 (1996), \#R2.

[8] B. Kupershmidt, q-Analogues of classical 6-periodicity, J. Nonlinear Math. Physics 10 (2003), 318-339.

[9] M. Shattuck, Bijective proofs of parity theorems for partition statistics, J. Integer Seq., 8 (2005), Art. 5.1.5.

[10] R. Stanley, Enumerative Combinatorics, Vol. I, Wadsworth and Brooks/Cole, 1986.

[11] C. Wagner, Generalized Stirling and Lah numbers, Discrete Math. 160 (1996), 199218.

[12] H. Wilf, generatingfunctionology, Academic Press, 1990. 